Cite this paper :

M Fenech, V Girod, V Claveria,

S Meance, M Abkarian, B

Charlot. Microfluidic blood

vasculature replicas using

backside lithography. Lab on a

Chip 19 (12), 2096-2106

DOI: $10.1039 /$ C9LC00254E

\section{Microfluidic blood vasculature replicas using backside lithography}

Marianne Fenech ${ }^{\mathrm{a}, \mathrm{c}}$, Vincent Girod ${ }^{\mathrm{b}}$, Viviana Claveriac ${ }^{\mathrm{c}}$, Sebastien Meance ${ }^{\mathrm{b}}$, Manouk Abkarianc, Benoit Charlot ${ }^{\mathrm{b}}$

Blood vessels in living tissues are an organized and hierarchical network of arteries, arterioles, capillaries, veinules and veins. Their sizes, lengths, shapes and connectivity are set up for an optimum perfusion of the tissues in which they deploy. In order to study hemodynamics and hemophysics of blood flows, but also to investigate artificial vasculature for organs on a chip, it is essential to reproduce most of these geometric features. Common microfluidic techniques produce channels with a uniform height and a rectangular cross-section that do not capture the sizes hierarchy observed in-vivo. This paper presents a new single-mask photolithography process using an optical diffuser to produce a backside exposure leading to microchannels with both a rounded cross-section and a direct proportionality between local height and local width, allowing a one step design of intrinsically hierarchical networks.

\section{Introduction}

Blood is a non-Newtonian complex fluid consisting of a dense suspension of cells in plasma. This set of cells is composed mainly of red blood cells (RBCs) but also in much smaller number, white blood cells and platelets. Due to its particulate nature, blood flow in vascular networks is complex and several remarkable properties are associated with it. First, blood is a shear-thinning fluid as quantified by Chien et al. ${ }^{1}$ in the late sixties. Blood viscosity drops when the shear rate increases. Two different regime of shear rates govern shear-thinning: when blood flows in large vessels, therefore at low shear rate, viscosity decrease is governed by the mechanisms of disassembly of large aggregates formed by RBCs due to depletion forces present in the plasma. On the other hand, when blood flows into smaller vessels and therefore at high shear rate, the deformability of the cells themselves become the major determinant of shear thinning ${ }^{2}$.

In addition, the Fåhræus-Lindqvist effect links the viscosity of the blood with the vessel diameter. This effect is due to the appearance of a cell layer in the vicinity of the walls where the shear rate is maximal and tends to concentrate cells in the center of the vessels. Thus, it lowers blood apparent viscosity when it flows into vessels down to $10 \mu \mathrm{m}$.

One of the keys to understanding hemophysics ${ }^{3}$ and blood flow dynamics is to build replicas of vascular networks with a geometry as close as possible to natural networks in order to observe the movements of RBCs under a microscope using high power illumination and a high-speed camera. Microfluidics and in particular microfluidic circuits made of PDMS have the ability to reproduce vascular networks that represent a model of blood microcirculation ${ }^{456}$. The transparency of PDMS as well as the micrometric size of patterns makes it possible to obtain replicas of vascularization that can be observed under a microscope.

\footnotetext{
a. Department of Mechanical Engineering, University of Ottawa, Ottawa, Canada.

b. Institut d'Electronique et des Systèmes IES, CNRS, University of Montpellier, Montpellier, France.

c. Centre de Biochimie Structurale CBS, CNRS, INSERM, University of Montpellier, France.
}

PDMS replicas of vascular network are an important tool for studying the distribution of RBC velocities $7,8,9$, cell deformations under high shear stresses ${ }^{2}$, oscillations of capillary blood flows $^{10}$, or the dynamics of RBCs aggregation ${ }^{11}$. Recent advances ar now integrating interactions between circulating cells and a model endothelium ${ }^{12,13,14}$ or polymer constructs that mimics the glycocalyx ${ }^{15}$. In addition to hemophysics studies, replicas of vascular network can be used to monitor the quality of stored blood ${ }^{16}$, as a diagnostic device for pathologies that affect blood rheology, to study the effect of cells deformability on the behavior of a blood circulation network ${ }^{17}$ and finally to simulate vascular anomalies ${ }^{18}$. These microfluidic circuits are two-dimensional projections of a network that is tridimensional by nature. Although such a projection in a plane cannot accurately represent the connectivity of a 3D network, this represents an approximation that may be acceptable if care is taken to maintain the section and length distributions of the vessels as well as the branching and loop statistics of a natural vascular network. This is clearly a disadvantage in terms of biomimetism but an advantage for microscopy.

Vascular networks have complex geometries made of interconnections, bifurcations and loops with vessels whose diameter varies dramatically (typically $4 \mathrm{~mm}$ down to $5 \mu \mathrm{m}$ ). The main function of this hierarchical network is to perfuse the tissues. The capillary network, also called capillary bed, is deployed in three dimensions in organs and tissues for optimal irrigation. Then, the venous network, out of the capillary bed harvests the cells that made the exchanges to bring them back to the heart. Recent progress in imagery allows now to reconstruct the exact topology of the vasculature of a whole $\operatorname{organ}^{19}$, including the capillary bed with thin capillaries.

After a short introduction on techniques developed for producing artificial vasculature, we report here an original technique that allows producing a microcirculation replica that mimics the geometry of natural vascular networks. The Microfabrication process presented here involves only one photomask, and uses the diffusion of UV light from the bottom of a negative photoresist layer to achieve a gradation of thickness from a single exposure. Microfluidic vascular networks produced with this technique are then used to observe particularities of blood flows. 


\section{Vasculature networks replicas}

Vascular networks have a specific geometry that depends on the organ where they deploy. However, we can observe common features such as tubular vessels, a distribution of vessels diameters between arteries, arterioles, capillaries, veinules and veins as well as the presence of loops. Several techniques have been developed so far to reproduce artificial networks with the specifications of transparency, surface roughness, minimum size around $5 \mu \mathrm{m}$, circular cross section and ease of fabrication.

Conventional photolithography. Replica moulding technologies using SU-8 photoresist on silicon and PDMS casting are the main techniques that have been employed for the fabrication of artificial networks. Although these techniques satisfy the specifications of transparency and micrometric precision, it does not faithfully reproduce the geometry of a vascular network. The main limitation concerns, in the first place, the fact that microfluidic circuits manufactured from a counter mould of a SU-8 photoresist, are composed of channels with rectangular sections but, above all, having all the same height. Conversely, a natural vascular network consists of vessels of more or less circular sections of different diameters showing a very wide range of diameter $(5 \mu \mathrm{m}$ to $4 \mathrm{~mm})$. When using standard photolithography techniques to reproduce a vascularization pattern, once can obtain a geometry similar to that shown in Figure 1(bottom riht) with uniform thickness and straight walls, which is far from the geometry shown in Figure 1 (bottom left).

Several research teams have then tried reproducing the complex geometry of vascular networks and in particular the specification of circular cross-sections. One of the first solution presented by Cokelet et al. in $1993^{20}$ is to use wet etching of glass substrates with hydrofluoric acid, allowing to obtain circular sections channels. Another solution is, for example, to first produce a microfluidic circuit of rectangular cross-sections, and then to introduce a non-crosslinked PDMS mixture to completely fulfill the channels. Finally, the injection of air under pressure creates a meniscus effect smoothing the channels cross-sections ${ }^{21,22}$. Although these techniques indeed make it possible to round the angles, they are complex to implement. Furthermore, they do not make it possible to obtain a gradation of the heights of the channels as a function of their widths. The laser ablation technique has also been investigated to directly write networks onto a polymer. If this technique allows indeed the production of multiple-level microfluidic channels ${ }^{23}$ it is at the expense of a relatively high surface roughness.

A simple solution for obtaining channels with a circular section is to anneal a thermoplastic positive photoresists ${ }^{29,30}$. It makes it possible to round the profile of channels as a function of surface tension when the viscosity of the polymer decreases with temperature. Depending on the wetting angle of the polymer on the substrate, it is even possible to approach semicircular sections. However, when the channel widths exceed a certain threshold the profile begins to resemble the section of a red blood cell.
3D printing techniques. Additive fabrication, and micro stereolithography technologies are currently under intense research efforts. These techniques have been employed successfully for the production of microfluidic devices but also in the field of microvasculature replicas ${ }^{24}$. One of the main advantages of these techniques is their ability to reproduce the actual 3D geometry of vascular networks. However, even though these techniques seem really promising they still do not have, for now, enough spatial resolution and sufficiently low surface roughness to be able to reproduce a capillary bed hierarchically connected to larger dendritic or reticulated networks. Finally, one technique that seems encouraging for the application described in this paper is the one developed by Lewis et al. ${ }^{25}$ using fugitive inks or liquid metals 26

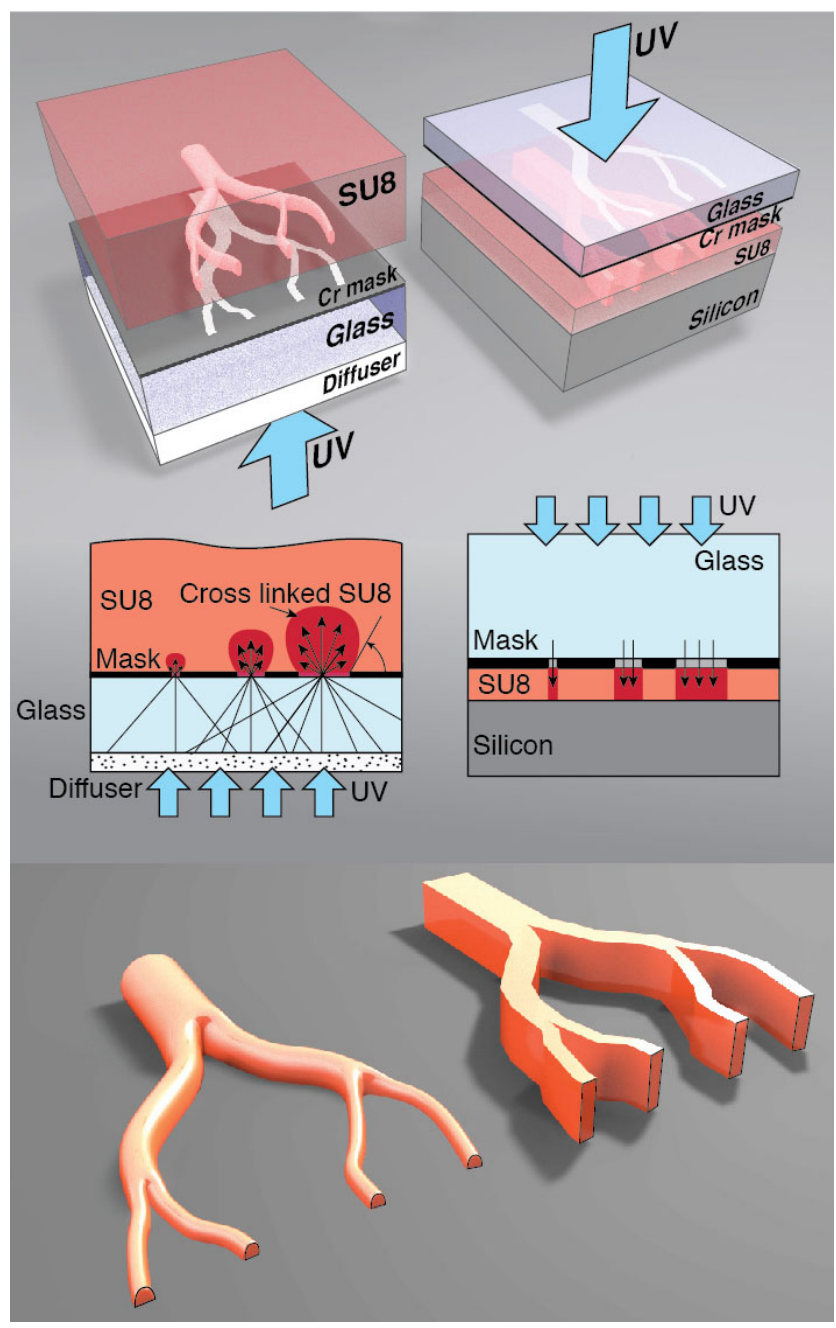

Fig. 1 Principle of the microfabrication processes for Artificial vascular networks. on the left side the principle of the proposed technique compared with, on the right side, standard topside photolithography, as well as 3D schematic of the geometries of vascular networks obtained with these techniques. 

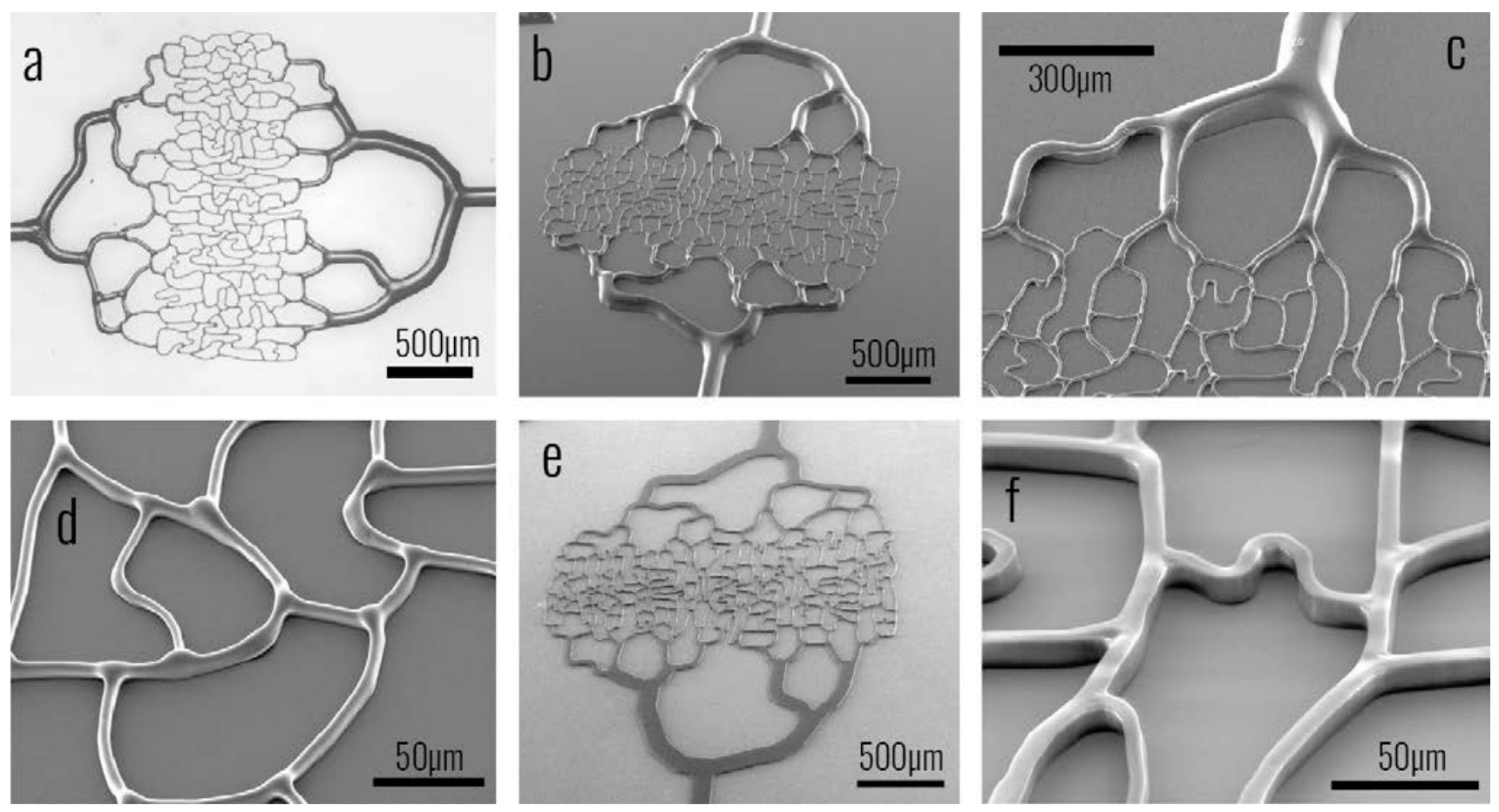

Fig. 2 Images of artificial vascular networks (a) Transmitted light optical photography (bright field) of the PDMS replica. (b,c,d) SEM image of the SU8 mould made with the backside lithography technique at different magnifications. We can observe the gradation in height according to width of channels. (e,f) SEM image of the same network made with a conventional top side exposed SU8 on silicon technique, we can observe the uniform thickness $(15 \mu \mathrm{m})$ and rectangular cross section.

\section{Backside illumination photolithography}

The solution we have chosen to explore in this work is backside lithography coupled to a light diffuser. This technique $31,32,33$ consisted in exposing a photoresist through a lithography mask, not pressed and on the surface of a resin-coated sample, as in conventional photolithography techniques, but located on the surface of a transparent substrate, as sketched in Figure 1. This solution therefore imposes an additional step, which is precisely the creation of the mask on the surface of the sample. This can be achieved either by deposition and engraving steps or by liftoff microstructuring on a glass substrate. Exposing a homogeneous resin thickness through the backside will lead to approximately the same resolution as a front-face lithography if the exposure doses are respected. The SU-8 negative photoresist, which is widely used in microfluidics, is very transparent in the $365 \mathrm{~nm}$ UV band. Therefore, a short exposure, just below the necessary doses makes it possible to cross-link large thicknesses of SU- 8 by eliminating at the same time the need of perfect contact between the lithography mask and the thick photoresist.

The use of a diffuser is the key element that allows height gradation as a function of width. The diffuser induces an effect that can be compared to micro-loading phenomena in plasma etching. An optical diffuser will thus diffuse the light from its surface according to its angular diffusion profile. The quality of the optical diffuser and in particular this angular diffusion profile is the critical parameter of this technique. Lee et al. ${ }^{31}$ showed that the profiles obtained in negative photoresists, mainly SU-8, are directly linked to a function of the polar diffusion intensity curves. They showed in particular that it was possible to obtain angles of up to $45^{\circ}$ between the photoresist and the substrate using an opal diffuser. Microfabrication results of an artificial vascular network produced with this technique are shown in Figure 2. We can notice, in particular the differences between this technique and the standard photolithography process (Figure 2(e,f)).

\section{Materials and Methods}

\section{Network design}

We designed an artificial network that is shown in Figure 2 and 4. It contains one inlet, one outlet and an arbitrary tree of microchannels of different widths that flare in tortuosities, bifurcations and loops. The geometry of this network was inspired from in-vivo networks but not exactly representative of a natural vascular network ${ }^{27}$ that followed the Murray's law ${ }^{28}$. Nevertheless, it has been designed to show a distribution of channels sizes from $120 \mu \mathrm{m}$ (inlet and outlet channels) down to $5 \mu \mathrm{m}$ (capillaries) that respected the statistical distribution ${ }^{27}$ of vessel sizes and lengths found in natural vascularizations. The inlet, mimicking an arteriole, was consecutively divided in daughter branches of smaller width with the following succession of widths: $120,75,35,22,12$ and finally $5 \mu \mathrm{m}$ for the capillaris. The capillary bed, unlike the arteriole tree, is made of several branches and loops. At the exit of the capillary bed, a network of veinules recovered the flows to concentrate them 
towards one unique outlet. This mask has been designed for the sole purpose of developing the technique presented here. The network spreads over a surface of $2.2 \times 3 \mathrm{~mm}$. The design has been made using Cadence Virtuoso. (Cadence Design Systems, Inc. San Jose, CA, USA).

\section{Microfabrication}

Substrate. The microfabrication process began with a $5 \times 5 \mathrm{~cm}$ and $500 \mu \mathrm{m}$ thick glass slides (Paul Marienfeld $\mathrm{GmbH}$, LaudaKönigshofen, Germany) that were previously cleaned with a piranha solution. Next, a $100 \mathrm{~nm}$ thick chromium layer was deposited on the whole surface by electron beam physical vapor deposition. A layer of AZ1518 photoresist (MicroChemicals, Ulm, Germany) is then spin-coated and prebaked at $110^{\circ} \mathrm{C}$ for $1 \mathrm{~min}$. The microcirculation pattern is transferred by contact photolithography, and the photoresist is developed in an alkaline solution. The mask is then defined by a wet etching process (chromium etching solution) resulting in a transparent network pattern. Finally, the remaining photoresist is stripped with acetone and oxygen plasma ashing. This chromium layer can be replaced by an Aluminum one, thick enough to be opaque to light with no consequence on the process.

Adhesion layer. A $1 \mu \mathrm{m}$ thick layer of SU-8 2002 (MicroChem, Westborough, MA, USA) is then spin-coated on the whole surface. The layer is pre-baked, flood exposed for 30s and baked at $95^{\circ} \mathrm{C}$ for $10 \mathrm{~min}$. This layer was used as a transparent adhesion layer for the forthcoming thick SU-8 layer that showed poor adhesion on glass substrates.

SU-8. A thick layer of SU-8 2010 was poured on the surface of the sample without spin-coating. In fact, since the exposure was made through the backside, a regular and perfectly flat layer of photoresist is not needed. The process only required the SU-8 thickness to be higher than the thicker channel to be made (about $150 \mu \mathrm{m}$ in our case). After $30 \mathrm{~min}$ relaxation, the thick SU-8 layer was ramped up ( 3 to $4^{\circ} \mathrm{C} / \mathrm{min}$ ) to $95^{\circ} \mathrm{C}$ and pre-baked for $30 \mathrm{~min}$ followed by a slow ramp down to room temperature. Due to thermal inertia of the hot plate, the ramp down to room temperature was more than one hour long. Other viscosities of SU-8 photoresist can be used, as long as the final thickness of the layer is higher than the thicker channel to be processed.

UV exposure. Exposure was then made through an opal diffuser (75mm opal diffuser \#46-662, Edmund Optics, Barrington, NJ, USA) in direct contact with the backside of the glass. The sample is placed upside down on a dark plastic sheet to avoid optical reflections. The proper UV dose $\left(\mathrm{mJ}^{\mathrm{cm}} \mathrm{cm}^{-2}\right)$ was optimized by trial and error method starting from the energy recommended by the manufacturer. Several exposure times were thus tested ranging from $5 \mathrm{~s}$ to $30 \mathrm{~s}$ using mercury vapor lamp and a longpass UV filter (PL-360LP, Omega Optical, Brattleboro, VT, USA) to eliminate UV radiation below $360 \mathrm{~nm}$. We found that the suitable exposure time was around $16 \mathrm{~s}$ for our masker, so an equivalent dose of $160 \mathrm{~mJ} . \mathrm{cm}^{-2}$ since our masker has an irradiance of $10 \mathrm{~mW} . \mathrm{cm}^{-2}$. This dose being usually appropriate for a $50 \mu \mathrm{m}$ thick SU-8 layer exposed trough conventional topside exposure.
Development and hard bake. Samples were then post-baked with a slow ramp up ( 3 to $4^{\circ} \mathrm{C} / \mathrm{min}$ ) to $95^{\circ} \mathrm{C}$ for $30 \mathrm{mn}$ and slowly ramped down to room temperature. Development was made in propylene glycol methyl ether acetate (PGMEA) until completion. This takes usually more than 30 min since the initial thickness of the resin was about several hundreds of microns. Finally, a hard-bake step was performed to soften the surfaces (ramp up to $160^{\circ} \mathrm{C}$ for 30 minutes followed by a slow cool down to room temperature).

\section{Microfluidic replicas}

PDMS microfluidic chips were fabricated with standard procedures. A 10:1 mix of PDMS and curing agents were prepared and mixed together. The mixture was then poured on the samples, degassed for 30 min under vacuum and cured at $80^{\circ} \mathrm{C}$ for two hours. Since SU-8 structures were rounded, showing negative clearance at some places, a silanization step was added to avoid attachment of PDMS on SU-8 and ease the unmoulding of the PDMS. It consisted in dipping the device for two hours in a hexane / (3-Aminopropyl) triethoxysilane (APTES) solution followed by an ethanol rinsing, $N_{2}$ drying and hotplate baking at $120^{\circ} \mathrm{C}$ for $20 \mathrm{~min}$, with slow temperature ramps. After dissection and extraction, PDMS circuits were then punched to create inlet and outlet, and intensely washed with DI water and ethanol. Finally, chips were treated by oxygen plasma (Plasmasystem Nano, Diener electronic $\mathrm{GmbH}$, Ebhausen, Germany) allowing a permanent bonding on a standard $170 \mu \mathrm{m}$ thick glass microscope slide.

Circuit preparation. Before blood flow experiments, PDMS circuit were hydrophylised using oxygen plasma in order to completely wet the capillary bed that can show high hydraulic resistance. Finally, a $0.1 \%$ bovine serum albumin was perfused for $10 \mathrm{mn}$ in the chip in order to prevent blood clogging.

\section{Microscopy and characterization}

Optical microscopy. Blood flow analysis was made using an inverted microscope (Zeiss axiovert 200, Carl Zeiss GmbH, Oberkochen, Germany) with several objectives ranging from $4 x$ to $100 x$. Images and movies were captured using a blue filter to enhance the contrast over RBCs and a camera fitted with a 2048×2048 pixels CMOS sensor (UI-3370CP, IDS Imaging Development Systems GmbH, Obersulm, Germany). Fast capture imaging was performed at frame rates from 60 up to $1500 \mathrm{fps}$ in order to observe individual cell movements.

Profilometry. Topology analyses were made using both mechanical and optical profilometers. Channels heights were measured using a Veeco Dektak 150 profiler (Veeco, Plainview, NY, USA) and images were taken using a Fogale zoomsurf optical profilometer (Fogale Nanotech, Nîmes, France).

SEM. Samples were analysed using FEI Inspect scanning electron microscope (ThermoFischer Scientific, Waltham, MA, USA) under Environmental SEM conditions, i.e at $0.8 \mathrm{mbar}$ pressure and with presence of vapour. Prior to this, samples were metallized with $100 \mathrm{~nm}$ Titanium using plasma sputtering. Hydrodynamic characterisation. The velocity of RBCs was estimated using particle image velocity (ImageJ plugging) as illustrated in Figure 6(c). The flow rate resulted from the product of the average velocity across the main arteriole (inlet 
vessel) by the cross-section area of the main arteriole. The overall network hydraulic resistance was then estimated as the ratio of the inlet pressure divided by the flow rate. In addition, the network flow distribution was investigated visually by manually counting the number of capillaries that were not flowing, named as "no flow". Finally, the local linear density of the RBC in the capillaries was estimated and reported as the average of RBC count aligned in $100 \mu \mathrm{m}$.

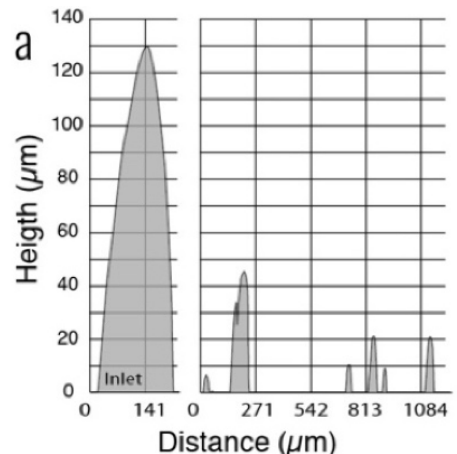

Distance $(\mu \mathrm{m})$

d
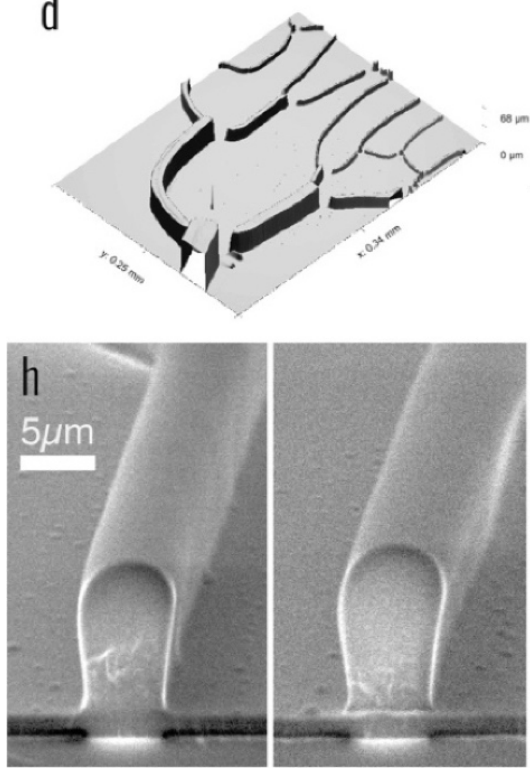
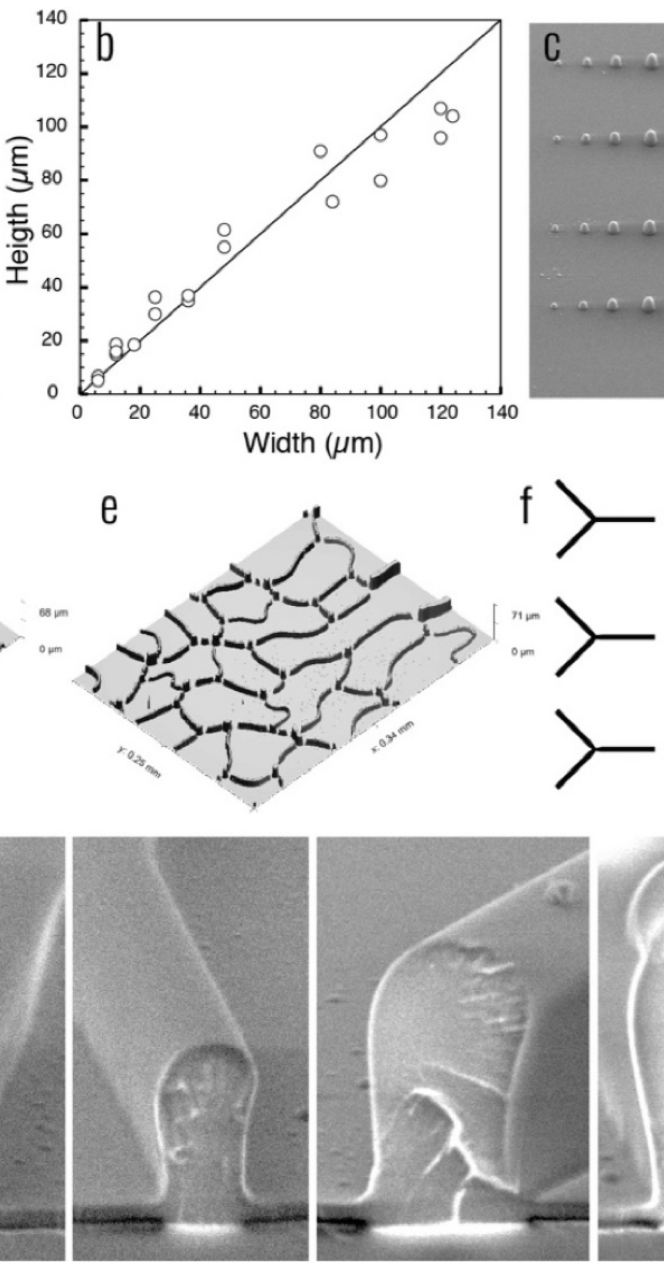
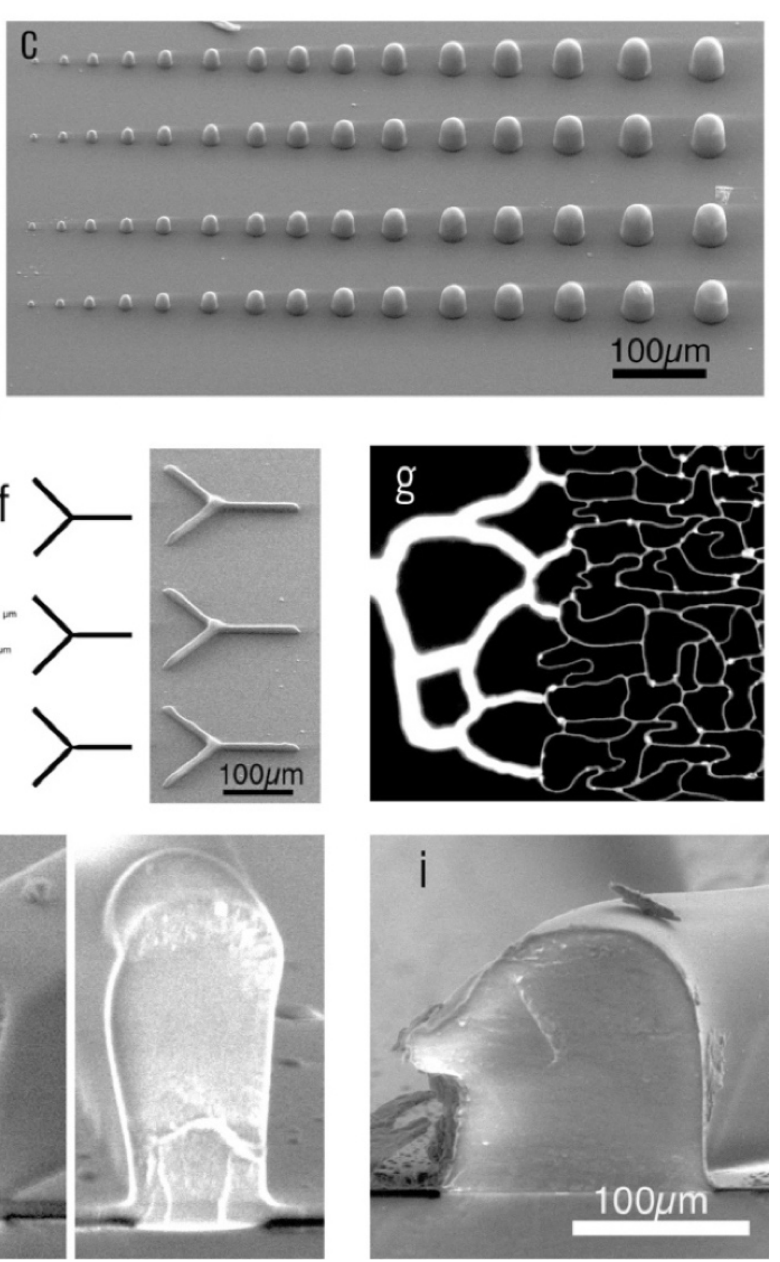

Fig. 3 Topography analysis of the network. (a) Profiles of the SU8 patterns measured with a Dektak profilometer along arbitrary lines perpendicular to the inlet-outlet axis, distribution of thicknesses ranges from 130 down to $5 \mu \mathrm{m}$. (b) Thickness of channels according to their nominal width on the photomask measured with the same technique. (c) SEM picture of test structures sowing an array of domes obtained with circular openings ranging from 8 to $36 \mu \mathrm{m}$ diameter $(\mathrm{d}, \mathrm{e})$ surface profile of different parts of the network made with optical interferometry. (f) Study of the effect of bifurcation shapes, bifurcation with continuously thick lines on the mask (top) shows bumps at node, the effect is reduced by thinning the lines at nodes (middle and bottom). ( $\mathrm{g}$ ) fluorescence measurement using rhodamine B as perfusing liquid, the intensity of fluorescence is proportional to channel thickness. (h) SEM images of cleaved SU-8 channels showing the cross section of 5 and $8 \mu \mathrm{m}$ capillaries and (i) for arteriole.

\section{Blood samples}

Non-clinically issued and venous blood samples for laboratories have been obtained from anomymous blood bags (that cannot be used for donation) through the local blood bank (Etablissement Français du Sang, Montpellier, France). All experiments were performed in compliance with the relevant laws and institutional guidelines. Whole blood was then centrifuged at $1000 \mathrm{~g}$ for $20 \mathrm{~min}$. Plasma and buffy coat, i.e the fraction of the centrifuged blood sample containing most of the white blood cells and platelets, were removed. RBCs were then washed 3 times in PBS, suspending the cells in the buffer solution and repeating the centrifugation and removal process. Afterward, cells were resuspended in an Optiprep (Sigma Aldrich, Ref. D1556) based solution, composed by $67 \%$ PBS and $33 \%$ Optiprep and complemented with $0.9 \mathrm{mg} / \mathrm{ml}$ of glucose. The use of Optiprep was made to match the typical mean density of RBC and avoid sedimentation in tubings. The final hematocrit was adjusted to $45 \%$. The entire process was done 
at room temperature. Blood suspension was then introduced in the microfluidic chip and flown using a microfluidic pressure controller (Elveflow OB1, Elvesys SAS, Paris, France). Experiments were done under pressures ranging from 1 up to 800 mbars. A total of four $1 \mathrm{ml}$ aliquots of blood suspension were used during the experiments on a dozen of microfluidic chips.

\section{Results and discussion}

\section{Microfluidic vascular replicas}

Topology. The microfabricated network, as observed by SEM imaging (secondary electrons, Figure $2(b, c, d)$ ) shows that the channels show a gradation of their thicknesses according to their widths. Thickness measurements obtained by a stylus profilometer are reported in Figure 3(a). It shows that within the scanned line, channels thicknesses are about $130 \mu \mathrm{m}$ for the inlet $(120 \mu \mathrm{m}$ initial width) and range from $50 \mu \mathrm{m}$ down to $5 \mu \mathrm{m}$ for arterioles and capillaries. However, this profilometry technique does not allow obtaining the exact profile of the section since this latter is convoluted by the conical shape and the velocity of the scanning probe. Figure 3(b) shows the thickness of channels according to their nominal width on the photomask, despite some relative variations it shows a rather linear evolution from 5 up to $130 \mu \mathrm{m}$.

Section. Blood vessels in-vivo show an almost circular cross section, so microfluidic replicas of vascular network should provide channels with a circular section. The backside illumination technique allows producing rounded sections, at least a section that is an image of the angular distribution of light intensity produced by the diffuser. In our case we found that indeed the cross section are rounded, as can be observe in cross sections of figure $3(\mathrm{~h}, \mathrm{i})$. This has to be compared with the channels made with standard frontside lithography of figure $2(e, f)$. This also can be observed in the test structure of Figure 3(c) that shows the results of circular openings in the mask ranging from 8 up to $36 \mu \mathrm{m}$ diameter. By observing the cross sections of capillary channels we can also notice the presence of a clearance angle that makes that the width of channels are larger on their top part than at the base. In order to obtain more circular cross section, as the ones obtained by Lee et al. ${ }^{31}$, one possibility would be to increase the exposure time but this requires also lowering the width of openings in the mask.

Roughness. We also noticed that the surface of the SU-8 channels showed some irregularities and a certain roughness. This was due to inhomogeneities of the light scattered from the backside. This roughness can be softened by a hard bake of the SU- 8 photoresist. Inlets and outlets were defined by $2 \mathrm{~mm}$ diameter circles and these wide openings created some rounded domes that receive the inlet and outlet tubings trough the PDMS slab. These large structures helped the introduction of blood samples in microchannels, avoiding clogging effects that often happen in conventional microfluidics.

Bumps at nodes. The backside illumination technology has some specificities regarding conventional lithography. Indeed, as we have shown before, the resulting height of microchannels was proportional to the width of the channels in the mask. When a bifurcation is drawn, the local surface is of higher dimension than the main part of the channels. This creates some bumps, or domes, at the location of bifurcations, as can be observed in SEM pictures of Figure 2(d) and in the fluorescence image of Figure $3(\mathrm{~g})$ where the intensity of fluorescence is proportional to the thickness. This effect can be reduced by locally thinning the line at bifurcations at the mask design level. This can observe in Figure $3(f)$ that shows three different designs of bifurcations with increasing level of local thinning (from top to bottom). The results show in the SEM picture of Figure 3(f) shows a lowering of the bump in bifurcations. 


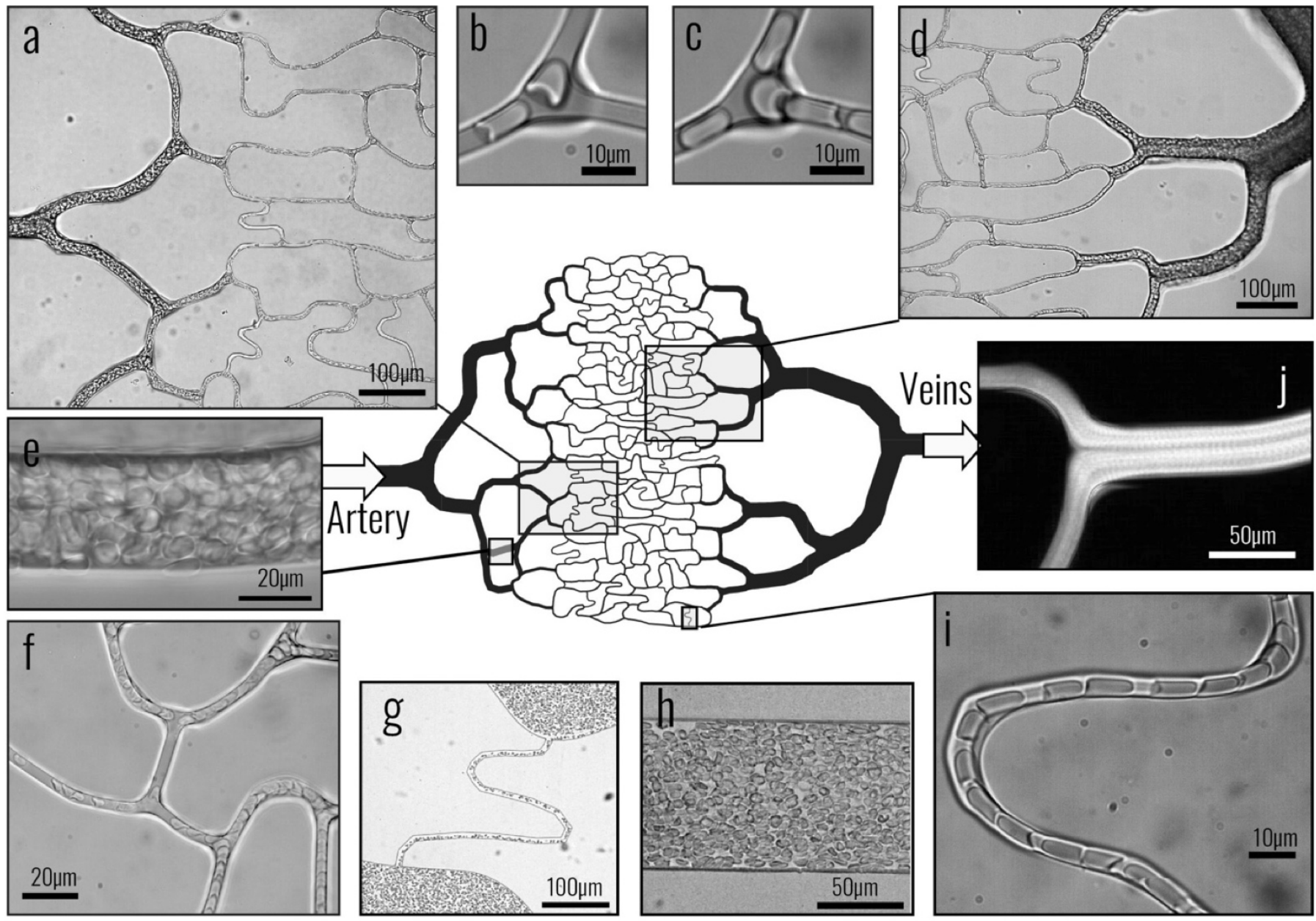

Fig.4 Videomicroscopy capture of blood flow experiments into artificial vascular network. (center) layout of the global circuit showing the network between the inlet and the outlet. (a,d) Arterioles and veinules parts of the network. (b,c) close up view of RBCs flowing in a bifurcation at high (200mbar) and low (10mbar) pressure, respectively. (e) Close up view of blood flow in arteriole showing the cell density and compaction. ( $f$ ) Close up view of RBCs flow in the capillary bed showing the difference of cell density in the capillaries. $(\mathrm{g}, \mathrm{h})$ Capture of blood flow in standard microfluidic circuit, e.g with $15 \mu \mathrm{m}$ constant thickness, within large and thin channels. We can notice that RBCs flow in the same plane, forming a single layer of cells. (i) Close up view of RBCs flowing in capillary under high inlet pressure (200mbar), we can observe the deformation of RBCs in a string of parachute shaped cells. (j) Superposition of video sequence frames (standard deviation) showing the local density of cells in a veinule bifurcation.

\section{Blood flow experiments}

Microfluidic circuits made with the fabrication process that we presented here have been used to observe some particularities of blood flows. Figure 4 shows some capture of $45 \%$ hematocrit blood flows in the network at different locations including arterioles, capillaries, bifurcations and veins.

\section{Comparisons with circuits of uniform height}

When comparing the distribution of RBCs flowing in our vascular network with blood flowing into circuits with uniform height (Figure $4(\mathrm{~g}, \mathrm{~h})$ ), the first observation that can be made is the difference of compaction of the cells. Indeed, as can be observed in Figure 4(e) that the arterioles are filled with high hematocrit blood and that the cells are strongly compacted without spaces between them. This should be compared to Figure $4(\mathrm{~g}, \mathrm{~h})$ in which blood flows in a standard microfluidic circuit with a uniform thickness of $15 \mu \mathrm{m}$, forming a single layer of cells and a spatial distribution in a single plane.

Another observation that can be made using these vasculature replicas is the fact that it contains thick and thin channels that are not in the same focal plane. This differs from standard microfluidic circuits where every channels are on the same plane. This can be observed in Figure 4(d) and video V2 showing that large and thick channels appear blurry compared to the thin ones because they are located outside the focal plane. Light absorption by the RBCs is also worth noting. In Figure 4(a,d), we can notice that blood flowing into large and thick arteries replica absorb a large part of the transmitted light, appearing darker than capillaries and thus making it difficult to observe and to spatially resolve all RBCs displacements, in contrary to those into slit like channels (Figure $4(\mathrm{~g}, \mathrm{~h})$ ). 


\section{Local hematocrit}

By observing the blood flow in channels with different sections, e.g cells in the capillary bed versus the arteriole or veinule part of the network (Figure 4(f) and video v2 for example), we can observe that cells are traveling the capillary bed with a lower hematocrit and that they have a different compaction compared with larger channels. This is called the network Fåhræus effect and it is caused by the fact that RBCs tend to move towards regions of low shear in the center of vessel. This has been observed in-vivo ${ }^{38}$ but also in microfluidic vascular networks ${ }^{39}$. We can also notice that some capillaries are "silent" for some input pressure conditions, meaning that a low number of cells are actually flowing in it. Figure 5(d) shows a measurement of the percentage of "silent" capillaries at the entrance and at the center of the capillary bed as function of the input pressure. We can notice that these percentages were not constant and changed with the input pressure. When the inlet pressure changes, the cell flow increases as well as the average cell velocity, the shear rate but also consequently the cell shape and finally the apparent viscosity of the blood. As a result, the distribution of pressures throughout the network is changed, which will induce local changes at the inlet and outlet of the capillary sections. For each input pressure value, a dynamic equilibrium will emerge and lead to different cell flows in each portion of the circuit and finally in some cases of silent sections.
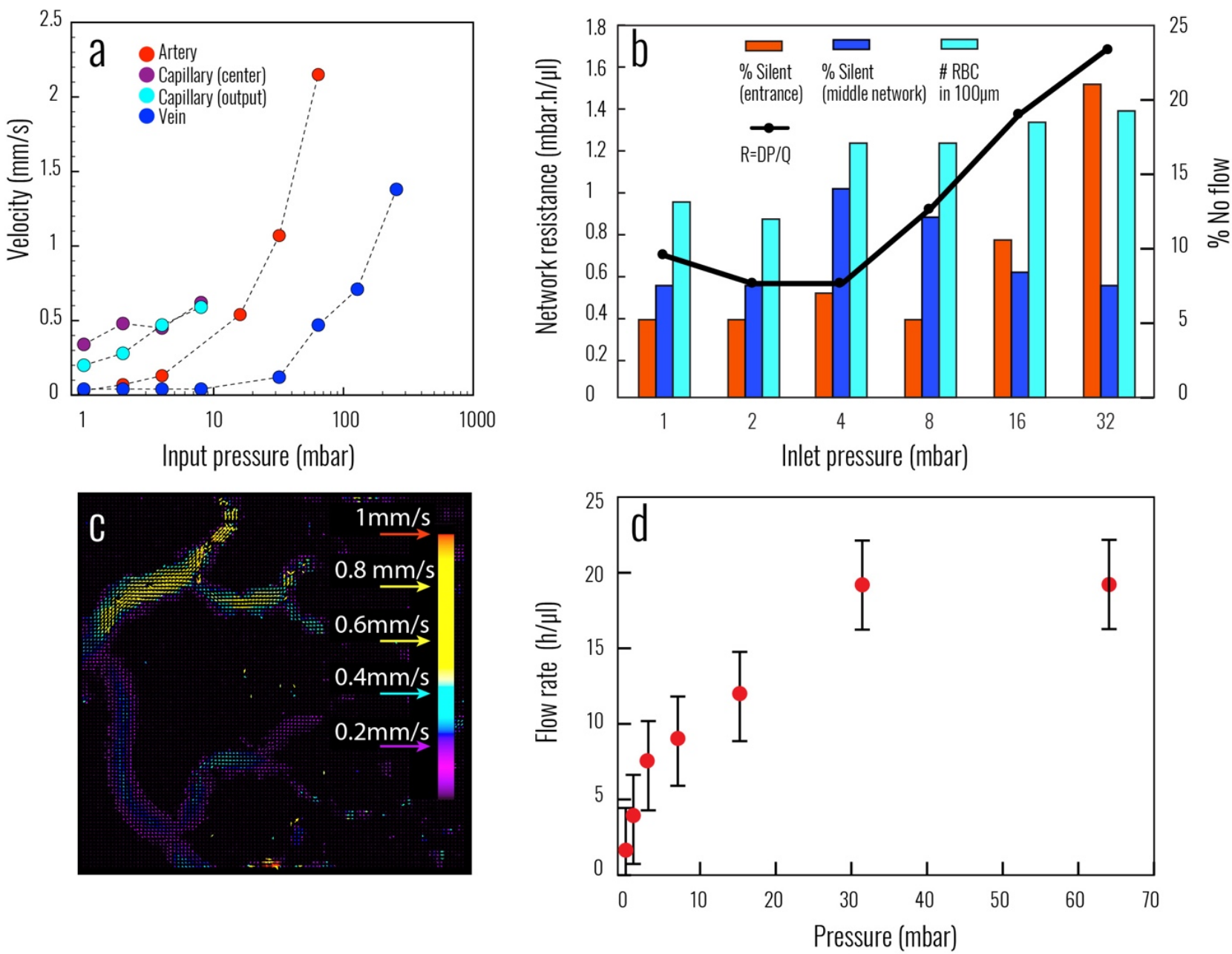

Fig.5 Flow and velocity analysis in the network. (a) Velocity of RBCs as function on input pressure at the inlet at different location of the network, artery, center capillary, output capillary and vein. (b) Hydraulic resistance of the network R=DP/Q as function of the inlet pressure. The red and blue bars report the percentage of capillary that are not flowing "\% no flow" at the entrance and in the center of the network respectively. The cyan bars present the linear red blood cell density in the capillary i.e. the average RBC count per $100 \mu \mathrm{m}$. (c) Example of Particle Image Velocimetry processing at the entrance of the network with an Input pressure of $16 \mathrm{mBar}$. (d) Total flow rate as function of the input pressure. Error bars present the uncertainty of the flow rate estimation due to an error of +/- 2 pixels/frame in the PIV measurement (which correspond to +/- $2.94 \mu \mathrm{l} / \mathrm{hr}$ ). 


\section{Journal Name}

RBC shape and deformation. RBC shape is strongly modified when cells arrive to small capillaries as is shown in Figure 4 (b,c, $f$ and i) and video 1 (Supplementary informations). Here RBCs adopt the typical parachute/bullet shape and their distribution is not homogeneous across the capillary. This effect is attributed to the hydrodynamic interaction between RBCs producing groups or train of cells. This effect has been studied in detail invitro $^{34-36}$ but also parachutes and cluster formation have been observed in-vivo experiments ${ }^{37}$. Our artificial network allows to mimic in a more accurate way a physiological capillary bed, were curvatures and bifurcations are always present.

Bifurcations. Figure $4(b, c)$ and video 1 (Supplementary informations) show the behaviour of RBCs crossing a bifurcation at high (200mbar) and low (10mbar) input pressure, respectively. We can first notice the change in shape of the cells that shows a parachute shape for high input pressure and discocyte shape for lower pressure. Due to the presence of bumps at nodes, the channel section at bifurcations increases and then we can observe some changes in the shape of cells, due to a relaxation of the cells when they pass through the bifurcation. This demonstrates the high deformability of the RBCs that adapt their shape to fit the dimensions of the vessel where they flow.

Cell free layer. For certain blood flow conditions, a cell-free layer (CFL) may appear near the walls of the vessel in which the blood flows ${ }^{41}$. This region depleted in cells is due to competition between hydrodynamic interactions between cells and wall, which tends to repel cells, and by cell-cell hydrodynamic interactions, which on the contrary tend to disperse RBCs. The size of this CFL is known to be in the range of one to two microns for $45 \% \mathrm{Ht}$ blood flowing in vessels from 10 to $50 \mu \mathrm{m}^{42,43}$, this value increasing as hematocrit decreases. This value of the CFL being small it has been difficult to observe it in our experiments. However, we found that in the veinule part of the network, a CFL was present, especially after the junction of thinner channels as can be observed in Figure 4(j). This effect is visible in the figure where we have superimposed about a hundred images taken in video by the standard deviation method on ImageJ. It can therefore be observed that the cells concentrate in the central part of the channel forming a CFL near the walls but also in between both cell tubes, this distribution continuing for a long distance after the bifurcation.

Velocity vs pressure. Particle image velocimetry technique has been used to extract the RBCs velocity in different parts of the network and for different input pressure. The results are shown in Figure 5(a) where the cell velocities have been recorded in arteries, at two locations of the capillary bed (center and near the output) and in the vein part of the network. We can first notice that the velocity of the cells increases more with the input pressure than in the arteries than in the veins. This is due to the fact that, by design, the vein network is slightly larger, on average, than the artery network. As shown in Figure 5(d), the flow rate does not increase linearly with an increase of pressure as it is expected for Newtonian flow in single channel. Therefore, the overall hydraulic resistance of the network is not constant as shown in Figure 5(b). Although these preliminary results do not allow to conclude with certainty on the origin of this non-linearity, it could be explained by the inhomogeneity of the flow repartition and the inhomogeneity of the cell density in the network. Indeed, when some capillaries are not perfused, it results in a higher flow rate in the other capillaries and the total length of the capillaries perfused decreases. In addition, the linear density of cells increases with the increase of input pressure as shown in Figure $5(\mathrm{~b})$. The increase of local cell density is likely linked with an increase of the local viscosity ${ }^{40}$ and therefore an increase of the hydraulic resistance.

\section{Conclusions}

This paper presented a novel microfabrication technique to produce artificial vascular networks whose geometry is close to natural vasculatures. The process is carried out by producing a SU-8 photoresist mould using a combination of backside illumination with an optical diffuser making it possible to obtain rounded cross section channels with a gradation of the thickness relative to their width with just one single mask. This new technology allows obtaining vascular networks that approach in vivo geometries for studying hemophysics and hemorheology. These circuits could also be used in the future as in imprint for epithelial cell culture in order to develop in vitro networks that could be the base of complex vascularised organ on chips ${ }^{43}$. This lithography technique can also be used in fields other than vascular networks, such as, for example, the shaping of branched hydrogel structures for mechanobiology or the capture of single cells. Finally, on the physical side these devices will be used to investigate more in detail the relations between flow in the network and inlet pressure, and specially the nonlinearities of hydraulic resistance we observed.

\section{Author contributions}

Conceptualization, B.C, V.G, M.F and M.A. Methodology, B.C, M.F, V.C and M.A. Microfabrication, B.C, V.G, and S.M. Experimental Investigations, B.C, V.C, M.F, S.M. Writing - review \& editing, B.C, V.C, M.F and M.A. Supervision, B.C. 


\section{Conflicts of interest}

"There are no conflicts to declare".

\section{Acknowledgements}

This work was supported by the LabEx NUMEV “Digital and Hardware Solutions, Environmental and Organic Life Modeling" (ANR-10-LABX-20-01) and by National Science and Engineering Research Council of Canada (\#RGPIN-2015-06188)

\section{Notes and references}

1. Chien, S. et al. Blood viscosity: influence of erythrocyte aggregation. Science157, 829-831 (1967).

2. Lanotte, L. et al. Red cells' dynamic morphologies govern blood shear thinning under microcirculatory flow conditions. Proc. Natl. Acad. Sci. 201608074 (2016).

3. Pries, A. R., Secomb, T. W., Gaehtgens, P. \& Gross, J. F. Blood flow in microvascular networks. Experiments and simulation. Circ. Res.67, 826-834 (1990).

4. Wong, K. H. K., Chan, J. M., Kamm, R. D. \& Tien, J. Microfluidic Models of Vascular Functions. Annu. Rev. Biomed. Eng.14, 205-230 (2012).

5. Sebastian, B., Dittrich, P. S. Microfluidics to Mimic Blood Flow in Health and Disease. Annu. Rev. Fluid Mech.50, 483-504 (2018).

6. Shevkoplyas, S. S., Gifford, S. C., Yoshida, T. \& Bitensky, M. W. Prototype of an in vitro model of the microcirculation. Microvasc. Res.65, 132-136 (2003).

7. Lima, R. et al. In vitro blood flow in a rectangular PDMS microchannel: experimental observations using a confocal micro-PIV system. Biomed. Microdevices10, 153-167 (2008).

8. Roman, S., Merlo, A., Duru, P., Risso, F. \& Lorthois, S. Going beyond $20 \mu \mathrm{m}$-sized channels for studying red blood cell phase separation in microfluidic bifurcations. Biomicrofluidics10, (2016).

9. Mehri, R., Laplante, J., Mavriplis, C. \& Fenech, M. Investigation of Blood Flow Analysis and Red Blood Cell Aggregation. 6

10. Forouzan, O., Yang, X., Sosa, J. M., Burns, J. M. \& Shevkoplyas, S. S. Spontaneous oscillations of capillary blood flow in artificial microvascular networks. Microvasc. Res.84, 123-132 (2012).

11. Fenech, M., Garcia, D., Meiselman, H. J. \& Cloutier, G. A Particle Dynamic Model of Red Blood Cell Aggregation Kinetics. Ann. Biomed. Eng.37, 2299-2309 (2009).

12. Lewis, D. M., Abaci, H. E., Xu, Y. \& Gerecht, S. Endothelial progenitor cell recruitment in a microfluidic vascular model. Biofabrication7, 045010 (2015).

13. Raasch, M. et al. Microfluidically supported biochip design for culture of endothelial cell layers with improved perfusion conditions. Biofabrication7, 015013 (2015).

14. Thuenauer, R., Rodriguez-Boulan, E. \& Römer, W. Microfluidic approaches for epithelial cell layer culture and characterisation. The Analyst139, 3206-3218 (2014).

15. Lanotte, L., Tomaiuolo, G., Misbah, C., Bureau, L. \& Guido, S. Red blood cell dynamics in polymer brush-coated microcapillaries: A model of endothelial glycocalyx in vitro. Biomicrofluidics8, 014104 (2014).

16. Burns, J. M., Yang, X., Forouzan, O., Sosa, J. M. \& Shevkoplyas,
S. S. Artificial microvascular network: a new tool for measuring rheologic properties of stored red blood cells. Transfusion (Paris)52, 1010-1023 (2012).

17. S. Shevkoplyas, S., Yoshida, T., C. Gifford, S. \& W. Bitensky, M. Direct measurement of the impact of impaired erythrocyte deformability on microvascular network perfusion in a microfluidic device. Lab. Chip6, 914-920 (2006).

18. Wu, Q. et al. Microfabrication of polydimethylsiloxane phantoms to simulate tumor hypoxia and vascular anomaly. J. Biomed. Opt.20, 121308 (2015).

19. Kelch, I. D. et al. Organ-wide 3D-imaging and topological analysis of the continuous microvascular network in a murine lymph node. Sci. Rep.5, 16534 (2015).

20. Cokelet, G. R., Soave, R., Pugh, G. \& Rathbun, L. Fabrication of in vitro microvascular blood flow systems by photolithography. Microvasc. Res.46, 394-400 (1993).

21. Yang, X., Forouzan, O., Burns, J. M. \& Shevkoplyas, S. S. Traffic of leukocytes in microfluidic channels with rectangular and rounded cross-sections. Lab. Chip11, 3231-3240 (2011).

22. Fiddes, L. K. et al. A circular cross-section PDMS microfluidics system for replication of cardiovascular flow conditions. Biomaterials31, 3459-3464 (2010).

23. Lim, D., Kamotani, Y., Cho, B., Mazumder, J. \& Takayama, S. Fabrication of microfluidic mixers and artificial vasculatures using a high-brightness diode-pumped Nd:YAG laser direct write method. Lab. Chip3, 318-323 (2003).

24. Li, X., Liu, L., Zhang, X. \& Xu, T. Research and development of 3D printed vasculature constructs. Biofabrication10, 032002 (2018).

25. Kolesky, D. B. et al. 3D bioprinting of vascularized, heterogeneous cell-laden tissue constructs. Adv. Mater. Deerfield Beach Fla26, 3124-3130 (2014).

26. Parekh, D. P., Ladd, C., Panich, L., Moussa, K. \& Dickey, M. D. 3D printing of liquid metals as fugitive inks for fabrication of 3D microfluidic channels. Lab. Chip16, 1812-1820 (2016).

27. Lipowsky, H. H. \& Zweifach, B. W. Network analysis of microcirculation of cat mesentery. Microvasc. Res.7, 73-83 (1974).

28. Barber, R. W. \& Emerson, D. R. Optimal design of microfluidic networks using biologically inspired principles. Microfluid. Nanofluidics4, 179-191 (2008).

29. Chen, Y.-C., Chen, G.-Y., Lin, Y.-C. \& Wang, G.-J. A lab-on-a-chip capillary network for red blood cell hydrodynamics. Microfluid. Nanofluidics9, 585-591 (2010).

30. Futai, N., Gu, W. \& Takayama, S. Rapid Prototyping of Microstructures with Bell-Shaped Cross-Sections and Its Application to Deformation-Based Microfluidic Valves. Adv. Mater.16, 1320-1323

31. Lee, J.-H., Choi, W.-S., Lee, K.-H. \& Yoon, J.-B. A simple and effective fabrication method for various 3D microstructures: backside 3D diffuser lithography. J. Micromechanics Microengineering18, 125015 (2008).

32. Lai, D. et al. Simple multi-level microchannel fabrication by pseudo-grayscale backside diffused light lithography. RSC Adv.3, 19467-19473 (2013).

33. Kang, M., Hwan Byun, J., Na, S. \& Li Jeon, N. Fabrication of functional 3D multi-level microstructures on transparent substrates by one step back-side UV photolithography. RSC Adv.7, 13353-13361 (2017).

34. Claveria, V. et al. Cluster of red blood cells in microcapillary flow: hydrodynamic versus macromolecule induced interaction. Soft Matter 12, 8235-8245, (2016). 
35. Tomaiuolo, G., Lanotte, L., Ghigliotti, G., Misbah, C., Guido, S. Red bloodcell clustering in poiseuille microcapillary flow. Physics of Fluids 24(5), 051903, (2012).

36. McWhirter, J. L., Noguchi, H., Gompper, G. Deformation and clustering of red blood cells in microcapillary flows. Soft Matter, 7(22), 10967-10977, (2011).

37. Skalak, R., Branemark, P. Deformation of red blood cells in capillaries. Science 9 164, 717-19, (1969).

38 Lipowsky, H.H., Kovalcheck, S., Zweifach, B.W. The distribution of blood rheological parameters in the microvasculature of cat mesentery. Circulation research 43, 738-749, (1978).

39. Reinhart, W. H., Piety, N. Z., Shevkoplyas, S. S. Influence of feeding hematocrit and perfusion pressure on hematocrit reduction (Fåhraeus effect) in an artificial microvascular network. Microcirculation 24(8), (2017).
40 Piety, N. Z., Reinhart, W. H., Stutz, J., Shevkoplyas, S. S. Optimal hematocrit in an artificial microvascular network, Transfusion 57(9), 2257-2266, (2017).

41 Kim, S. Ong, P.K., Yalcin, O., Intaglietta, M., Johnson, P.C. The cell-free layer in microvascular blood flow. Biorheology 46(3), 181-9, (2009).

42. Fedosov, D.A., Caswell, B., Popel, A. S., Karniadakis, G. E. M. Blood Flow and Cell-Free Layer in Microvessels. Microcirculation 17(8), (2010).

43 Bento, D., Pereira, A. I., Lima, J., Miranda, J. M., Lima, R. Cellfree layer measurements of in vitro blood flow in a microfluidic network: an automatic and manual approach. Comput. Methods Biomech. Biomed. Eng. Imaging Vis. 6 629-37, (2018)

43 Tsvirkun, D., Grichine, A., Duperray, A., Misbah, C., Bureau, L. Microvasculature on a chip: study of the Endothelial Surface Layer and the flow structure of Red Blood Cells. Scientific Reports 7, 45036, (2017) 